\title{
Complexity and Hopf Bifurcation Analysis on a Kind of Fractional-Order IS-LM Macroeconomic System
}

\author{
Junhai Ma* and Wenbo Ren ${ }^{\dagger}$ \\ Nonlinear Dynamics and Chaos Group, \\ College of Management and Economics, \\ Tianjin University, Tianjin 300072, P. R. China \\ *mjhtju@aliyun.com \\ †rw120912@126.com
}

Received January 29, 2016; Revised May 1, 2016

\begin{abstract}
On the basis of our previous research, we deepen and complete a kind of macroeconomics ISLM model with fractional-order calculus theory, which is a good reflection on the memory characteristics of economic variables, we also focus on the influence of the variables on the real system, and improve the analysis capabilities of the traditional economic models to suit the actual macroeconomic environment. The conditions of Hopf bifurcation in fractional-order system models are briefly demonstrated, and the fractional order when Hopf bifurcation occurs is calculated, showing the inherent complex dynamic characteristics of the system. With numerical simulation, bifurcation, strange attractor, limit cycle, waveform and other complex dynamic characteristics are given; and the order condition is obtained with respect to time. We find that the system order has an important influence on the running state of the system. The system has a periodic motion when the order meets the conditions of Hopf bifurcation; the fractional-order system gradually stabilizes with the change of the order and parameters while the corresponding integer-order system diverges. This study has certain significance to policymaking about macroeconomic regulation and control.
\end{abstract}

Keywords: Fractional-order; IS-LM model; Hopf bifurcation; complexity; chaos attractor.

\section{Introduction}

In recent years, the theory of nonlinear dynamics, especially chaos theory, has become an important tool in the analysis of economic and management system. More and more scholars have achieved good results in this field. Since the economic system is complex and changeable, the nonlinear dynamic theory, including chaos theory, plays an important role in the aspect of the internal evolution of the system. Besides, chaos theory has been applied to various fields of economic and management, and achieves good results. These include the van der Pol [Chian et al., 2006] model, Kaldorian model [Lorenz, 1993] and Goodwins accelerate model [Lorenz \& Nusse, 2002], etc.

At present, the main research of nonlinear theory in the economics sphere is to analyze the actual operation of the market with corresponding economic models. These models are based on chaos theory, game theory, and others. As shown by Ma and Zhang [2012], in the oligopoly insurance market, the complex dynamic characteristics of the system will be changed when the decision of the oligopoly is delayed. Li and Ma [2013b] focused on three

\footnotetext{
*Author for correspondence

This is an Open Access article published by World Scientific Publishing Company. It is distributed under the terms of the Creative Commons Attribution 4.0 (CC-BY) License. Further distribution of this work is permitted, provided the original work is properly cited.
} 
oligopoly markets with R\&D competition, and analyzed the complex dynamic characteristics, which shows the major role of $R \& D$ in the market competition.

At the beginning, fractional-order calculus theory was applied to physics analysis. As the study is deepened gradually, more and more experts and scholars found that fractional-order theory is close to actual economics and management. Furthermore, it is a good reflection on the memory characteristics of economic variables; therefore, it has been widely used in recent years, achieving good results, such as the Duffing system [Ge \& Ou, 2007; Sun et al., 2009], the van der Pol model [Barbosa et al., 2007; Chen \& Chen, 2008; Ma et al., 2006; Chian et al., 2006], as well as the Chen system model [Ma \& Chen, 2001a, 2001b; Ahmed et al., 2006].

As a classic economics model, the IS-LM model is representative in the macroeconomic analysis. In recent years, many scholars carried out research on nonlinear dynamics and chaos theories, and achieved fruitful results [Schinasi, 1982; Cai, 2005; Cesare \& Sportelli, 2005; Milhaela et al., 2007]. Since the economic system is a nonlinear complex system, the research in this area is gradually increasing to include studies of Hopf bifurcation [Li \& Wu, 2014; Liu et al., 2014], complex dynamical characteristics of different economic systems [Zhang \& Wei, 2004; Szydlowski \& Krawiec, 2005; Tu \& Ma, 2014; Gao \& Ma, 2009; Ma \& Gao, 2007], as well as fractional-order theory in the economic system [Li \& Ma, 2013a; Tavazoei et al., 2009b; Tavazoei et al., 2009a].

In the course of this study, we found that there is almost no research on the expansion of IS-LM model with fractional-order theory, so we deepen and complete the analysis on the traditional ISLM model with fractional-order theory, and discuss the evolution trend and complex dynamics characteristics in the fractional-order system, explore the internal features, and analyze the Hopf bifurcation conditions and the impact of parameters' adjustment to the system.

\section{Preliminaries}

Generally speaking, there are mainly three kinds of definitions of fractional-order calculus, including respectively the Riemann-Liouville definition [Ross, 1975], Grünward-Letnikov definition [Oldham \& Spanier, 1974] and Caputo definition [Podlubny, 1998]. Among them, the Riemann-Liouville definition is mainly adopted in the related theories and mathematical analysis of fractional-order calculus [Mao et al., 2004; Li et al., 2005]. But, in actual application, the Caputo definition can be understood as an weighted integral of the integerorder derivative in an interval time, which has a good effect on the definition of the boundary conditions, so it is widely used in physics. In the analysis of economics, the Caputo definition provides a good explanation about the memory characteristics of economic variables, so it is widely applied in various economic models. Here, we adopt the Caputo fractional derivative, which is defined as follows.

\subsection{Definition of Caputo fractional derivative}

$$
{ }_{q}^{C} D_{t}^{q} f(t):=\left\{\begin{aligned}
\frac{1}{\Gamma(n-q)} \int_{q}^{t} & \frac{f^{(n)}(\tau)}{(t-\tau)^{q+1-n}} d \tau, \\
& n-1<q<n \\
\frac{d^{n}}{d t^{n}} f(t), & q=n
\end{aligned}\right.
$$

where $a \leq t \leq b$, and $q$ is the order of Caputo derivative, denoted as: $D_{t}^{q} f(t)$ and $\Gamma$-function $\Gamma(n-q)=$ $\int_{0}^{\infty} e^{-t} t^{n-q-1} d t$

\subsection{Stability analysis}

Fractional-order system has the same equilibrium point with the corresponding integer-order system, but the stability conditions are different, even if the real part of the eigenvalue is positive, the equilibrium point of fractional-order system may still be stable.

Lemma 1 [Stability Condition of FractionalOrder System]. Linear autonomous system $D^{\alpha} x(t)=A(x)$, where $A$ is $n \times n$ coefficient matrix, the system is asymptotically stable at the equilibrium point if and only if: $\left|\arg \left(\operatorname{eig}(A)_{i}\right)\right|>$ $\max \left[\alpha_{i} \pi / 2\right]$, this is a sufficient condition for asymptotic stability of the equilibrium point; accordingly, the necessary condition of equilibrium point entering into chaos: $\min _{i}|\arg (\operatorname{eig}(A))| \leq \min _{i}\left[\alpha_{i} \pi / 2\right]$.

\subsection{Numerical algorithms}

In the fractional-order system, the main idea is to discretize and calculate the approximate solution. 
The most widely used method is Adams-BashforthMoulton scheme which has higher precision and smaller error; we also adopt it in this paper.

Consider the fractional differential equation:

$$
\begin{array}{r}
D^{q} y(x)=f(t, y(t)), \quad a \leq t \leq b, \quad y^{(k)}(0)=y_{0}^{(k)}, \\
k=0,1, \ldots, m-1 \quad \text { and } \quad m=\lceil q\rceil .
\end{array}
$$

It is equivalent to the Volterra equation:

$y(t)=\sum_{k=0}^{m-1} y_{0}^{(k)} \frac{t^{k}}{k !}+\frac{1}{\Gamma(q)} \int_{0}^{t}(t-s)^{q-1} f(s, y(s)) d s$.

According to Adams-Bashforth-Moulton scheme, we can export the expression of predictorcorrector algorithm.

\section{IS-LM Model and Corresponding Fractional-Order System}

IS-LM model is a classic economics model which has very good effect on the relationship among various elements, based on the efforts of many scholars, the differential equation of IS-LM model has made much progress. For a more economic model, we consider the following fixed-price disequilibrium intermediate run IS-LM model augmented by a government budget constraint.

$$
\left\{\begin{array}{l}
\dot{y}(t)=\alpha\left[I(y(t), r(t))+g-S\left(y^{D}(t)\right)-T(t)\right] \\
\dot{r}(t)=\beta[L(y(t), r(t))-m(t)] \\
\dot{m}(t)=g-T(t) .
\end{array}\right.
$$

Here, $y(t)$ is income; $I(Y(t), R(t))$ are investment; $g$ is government expenditure; $S\left(y^{D}(t)\right)$ is savings which are related to the disposable income $y^{D}(t) ; T(t)$ is tax revenue; $r(t)$ is interest rate; $L(Y(t), R(t))$ are demand for money; $m(t)$ is real currency supply quantity, $\alpha_{i}, \beta_{i}(i=1,2)$ are positive parameters representing the speed changes of the income and interest rates respectively.

In the traditional IS-LM macroeconometric model, when equilibrium occurs in the goods and monetary markets, the model is a two-dimensional model. On combining with Kaldor-Kalecki business cycle, scholars constructed the three-dimensional IS-LM macroeconomics model. Under the condition that the international trade is not considered, the constructed model reflects the relationship of macroeconomic variables well, and it is also in line with the static IS-LM model. We can see that, in the process of complex dynamics analysis with the variables: income $y(t)$, interest rate $r(t)$ and real currency supply quantity $m(t)$, investment, demand for money, savings and tax revenue are the most important variables. They all have a connection with $y(t), r(t), m(t)$. This is also the reason we need to abandon the static model and have the dynamics to analyze the macroeconomic system.

Based on the research of differential IS-LM model, we consider the following specific functions:

Investment

$$
I(Y(t), R(t))=\frac{a^{y(t)}}{r(t)} .
$$

Demand for money

$$
L(Y(t), R(t))=m Y(t)+\frac{l_{1}}{r(t)-l_{2}},
$$

$l_{1}, l_{2}>0$ and $l_{2}$ is a fixed very small rate of interest generating the liquidity trap.

Savings

$$
S\left(y^{D}(t)\right)=s(1-\varepsilon) y(t), \quad 0<s<1 .
$$

Tax revenue

$$
T(t)=\varepsilon y(t) .
$$

In order to guarantee the actual simulation to the macroeconomic system, and make the model more general, improve the differential equations model into fractional-order model, therefore, the model can be expressed as:

$$
\left\{\begin{array}{l}
D^{q}(y)=\alpha\left[\frac{a^{y(t)}}{r(t)}+g-s(1-\varepsilon) y(t)-\varepsilon y(t)\right] \\
D^{q}(r)=\beta\left[m y(t)+\frac{l_{1}}{r(t)-l_{2}}-m(t)\right] \\
D^{q}(m)=g-\varepsilon y(t) .
\end{array}\right.
$$

The model changes in commensurate to order $q$, and the first equation represents the equilibrium of the product market; the second equation represents the equilibrium of monetary market.

\subsection{Model analysis}

Solving the equilibrium point of system (2), we can easily obtain that system (2) has only one 
equilibrium point: $E_{0}=\left(y_{0}, r_{0}, m_{0}\right)$, where:

$$
y_{0}=\frac{g}{\varepsilon}, \quad r_{0}=\frac{a}{s(1-\varepsilon)}, \quad m_{0}=\frac{\varepsilon g m s l_{2}-\varepsilon^{2} s l_{1}-g m s l_{2}+a g m+\varepsilon s l_{1}}{\varepsilon\left(\varepsilon s l_{2}-s l_{2}+a\right)} .
$$

So, $E_{0}$ is the only Nash equilibrium point, when $y_{0}, r_{0}, m_{0}>0, E_{0}$ non-negative and with economic significance.

For the convenience of calculation, move the equilibrium point to the origin.

$$
Y(t)=y(t)-y_{0}, \quad R(t)=r(t)-r_{0}, \quad M(t)=m(t)-m_{0} .
$$

Then the system (2) changes into:

$$
\left\{\begin{aligned}
D^{q}(Y)= & \frac{\alpha}{\varepsilon(R \varepsilon s-R s-a)}\left(R Y \varepsilon^{3} s^{2}-R Y \varepsilon^{3} s-2 R Y \varepsilon^{2} s^{2}+R \varepsilon^{2} g s^{2}\right. \\
& \left.+R Y \varepsilon^{2} s+R Y \varepsilon s^{2}-2 R \varepsilon g s^{2}+R g s^{2}+Y a \varepsilon^{2}\right) \\
D^{q}(R)= & \frac{\beta}{\left(R \varepsilon s-\varepsilon s l_{2}-R s+s l_{2}-a\right)\left(\varepsilon s l_{2}-s l_{2}+a\right)}\left(R Y \varepsilon^{2} m s^{2} l_{2}-Y \varepsilon^{2} m s^{2} l_{2}^{2}-2 R Y \varepsilon m s^{2} l_{2}\right. \\
& -R M \varepsilon^{2} s^{2} l_{2}+2 Y \varepsilon m s^{2} l_{2}^{2}+M \varepsilon^{2} s^{2} l_{2}^{2}+R Y a \varepsilon m s+R Y m s^{2} l_{2}+2 R M \varepsilon s^{2} l_{2}+R \varepsilon^{2} s^{2} l_{1} \\
& -2 Y a \varepsilon m s l_{2}-Y m s^{2} l_{2}^{2}-2 M \varepsilon s^{2} l_{2}^{2}-R Y a m s-R M a \varepsilon s-R M s^{2} l_{2}-2 R \varepsilon s^{2} l_{1} \\
& \left.+2 Y a m s l_{2}+2 M a \varepsilon s l_{2}+M s^{2} l_{2}^{2}+R M a s+R s^{2} l_{1}-Y a^{2} m-2 M a s l_{2}+M a^{2}\right) \\
D^{q}(M)= & -Y \varepsilon .
\end{aligned}\right.
$$

Therefore, the research on the stability of the equilibrium point $E_{0}$ changes into the stability of the origin of system (3).

Calculate the Jacobi matrix, at the origin, the Jacobi matrix $J$ as follows:

$$
J=\left[\begin{array}{ccc}
-\alpha \varepsilon & -\frac{\alpha g s^{2}(-1+\varepsilon)^{2}}{a \varepsilon} & 0 \\
\beta m & -\frac{\beta l_{1} s^{2}(-1+\varepsilon)^{2}}{\left(\varepsilon s l_{2}-s l_{2}+a\right)^{2}} & -\beta \\
-\varepsilon & 0 & 0
\end{array}\right] .
$$

Calculate the eigenvalue of the Jacobi matrix, the characteristic equation is:

$$
P(\lambda)=\lambda^{3}+a_{1} \lambda^{2}+a_{2} \lambda+a_{3}=0
$$

where:

$$
\begin{aligned}
& a_{1}=\alpha \varepsilon+\frac{\beta l_{1} s^{2}(\varepsilon-1)^{2}}{\left(\varepsilon s l_{2}-s l_{2}+a\right)^{2}} \\
& a_{2}=\frac{\alpha \beta l_{1} \varepsilon s^{2}(\varepsilon-1)^{2}}{\left(\varepsilon s l_{2}-s l_{2}+a\right)^{2}}+\frac{\alpha \beta g m s^{2}(\varepsilon-1)^{2}}{a \varepsilon} \\
& a_{3}=\frac{\alpha \beta g s^{2}(\varepsilon-1)^{2}}{a} .
\end{aligned}
$$

According to the Routh-Hurwitz criterion, we know that the necessary and sufficient conditions for the stability of the nonlinear system (3) are: $a_{1}>0, a_{2}>0, a_{3}>0$ and $a_{1} a_{2}-a_{3}>0$, and according to chaos theory, if all the equilibrium points of a system are asymptotically stable, the self-excited attractor does not exist, the solution of the system will be asymptotically stable to a fixed value, and cannot form the basin of the attraction. The self-excited attractor of an unstable system can gradually appear with the iterative of the trajectory, and remain in oscillation state.

Solve the roots of characteristic equation $P(\lambda)$, that is, the eigenvalues of the Jacobi matrix $J$. According to Lemma 1, $m\left(q^{*}\right)=q^{*} \pi / 2-\min \times$ $\left|\arg \left(\lambda_{i}\right)\right|=0$, we should calculate the characteristic equation and eigenvalues of the system (3), and make the judgment of the fractional-order Hopf bifurcation of order conditions of the system. Due to the system (3) being large and having too many parameters, the expression of the characteristic equation and eigenvalue is too cumbersome, this is no longer listed. In the numerical simulation process, we can first make the parameter assignment, and calculate the characteristic values of the system, determine the conditions for Hopf bifurcation and the stability of the system. 


\subsection{Experimental simulation calculation}

In order to reflect the dynamic behavior and dynamic characteristics of the system, numerical simulation and analysis are adopted in the following text. In the simulation process of fractional-order systems, we adopt Adams-Bashforth-Moulton scheme; and calculate the integer-order differential system with the original Runge-Kutta method. Identify the order of the fractional-order system by Lemma 1 when Hopf bifurcation occurs, then determine the effect of parameter variation on the running state of the fractional-order and the integer-order system.

First of all, the parameters assigned to the system are:

$$
\begin{aligned}
& a=0.37, \quad \alpha=0.95, \quad \beta=0.9, \\
& l_{1}=1, \quad l_{2}=0.0075, \quad m=0.0035, \\
& g=30, \quad s=0.25, \quad \varepsilon=0.01 .
\end{aligned}
$$

The equilibrium point of system (3) is:

$$
\begin{gathered}
E_{0}=\left(y_{0}=3000, r_{0}=1.494949495,\right. \\
\left.m_{0}=11.17229173\right) .
\end{gathered}
$$

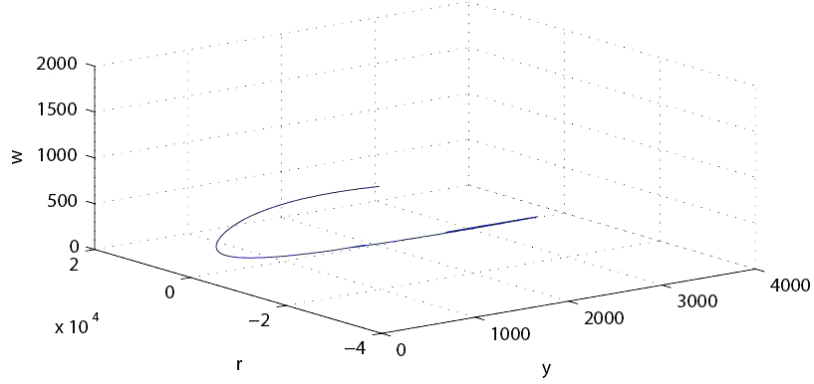

(a)

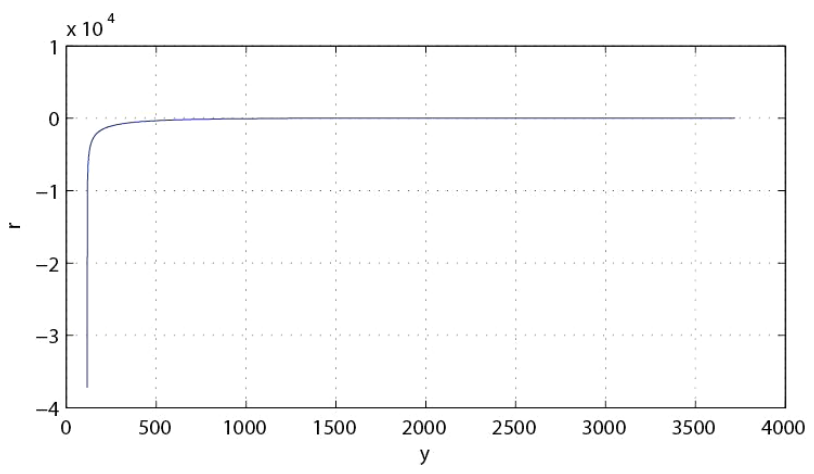

(c)
Translation transform $Y(t)=y(t)-y_{0}, R(t)=$ $r(t)-r_{0}, M(t)=m(t)-m_{0}$, then the Jacobian matrix and eigenvalue of the system (3) are respectively:

$$
\begin{aligned}
J= & {\left[\begin{array}{ccc}
-0.0095 & -471.8386825 & 0 \\
0.00315 & -0.4067785578 & -0.9 \\
-0.01 & 0 & 0
\end{array}\right] } \\
\lambda_{1} & =-1.43660468, \\
\lambda_{2,3} & =0.51016306 \pm 1.64185723 i .
\end{aligned}
$$

At this moment, the characteristic equation of the system is:

$$
P(\lambda)=\lambda^{3}+0.4163 \lambda^{2}+1.65527 \lambda+4.24629=0 .
$$

According to the Routh-Hurwitz criterion, we can obtain the stability conditions for the nonlinear system (3): $a_{1}>0, a_{2}>0, a_{3}>0$, however, $a_{1} a_{2}<$ $a_{3}$, the equilibrium point of the system is unstable.

The order of fractional-order system has a great influence on the running state, which can change the running state and the dynamic characteristics of the system frequently, in the fractional-order ISLM model, when the order of $q$ is equal to 0.2 , the system forms a chaos attractor, as shown in Fig. 1.

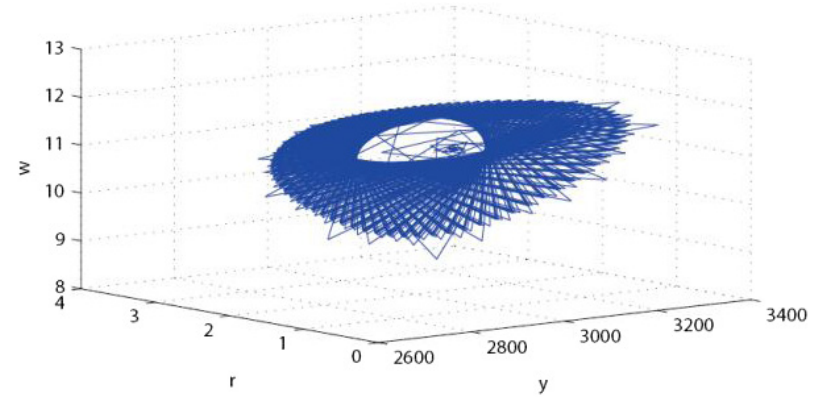

(b)

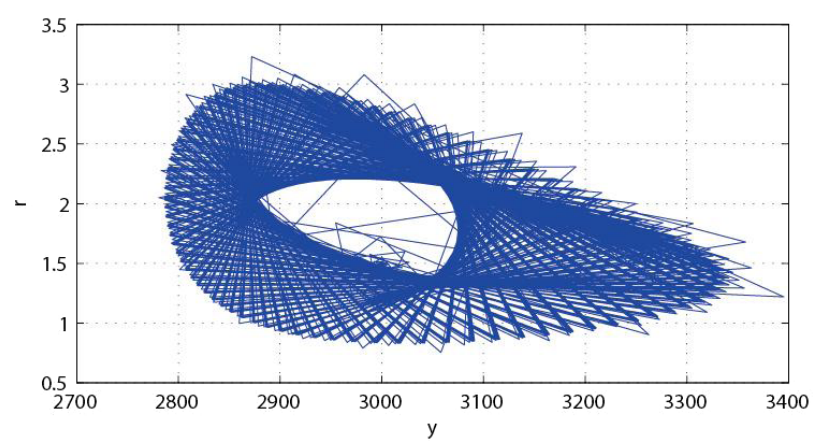

(d)

Fig. 1. Running state and the chaotic attractor when the order $q=1,0.2$. (a) Running state when $q=1$, (b) chaotic attractor when $q=0.2$, (c) projection on the $y-r$ plane and (d) projection on the $y-r$ plane. 
As shown in Fig. 1, the order $q$ of system (2) has a considerable influence on the operation of the system state. Under the integer-order $(q=1)$ conditions, the system operates smoothly, and converges to 116.5 , which is much smaller than the initial value, as shown in Figs. 1(a) and 1(c).

As the order of $q$ decreases, the system enters into the fractional-order state, and the running state of the system gradually changes, this paper presents the chaotic attractors of the system (2) occuring when $q=0.2$, as shown in Figs. 1(b) and $1(\mathrm{~d})$, at this time, although the system is in a chaotic state, the chaotic attractor ensures the solutions are fixed in the phase space.

Therefore, this paper presents the running state of the system with the change of the order $q$, as shown in Fig. 2.

It can be seen that when the order is within the interval of $0.235-0.81$, the national income is stable at the point of 3000 . The national income will reduce rapidly with the increase of the order, which is about 116.5. This is not allowed in the actual economic system. So in the actual operation of the economic system, the order should be maintained in the range of $(0.235,0.81)$.

In the actual economic system, the order of the system is usually kept within $(0,1)$. In order for a further effect of order on the running state of the system, we expand the scope of the order to 2 , and we can see that, when the order of the system is gradually increased to 1.825 , the system will produce a chaotic state. The economic system will be no longer stable and controllable.

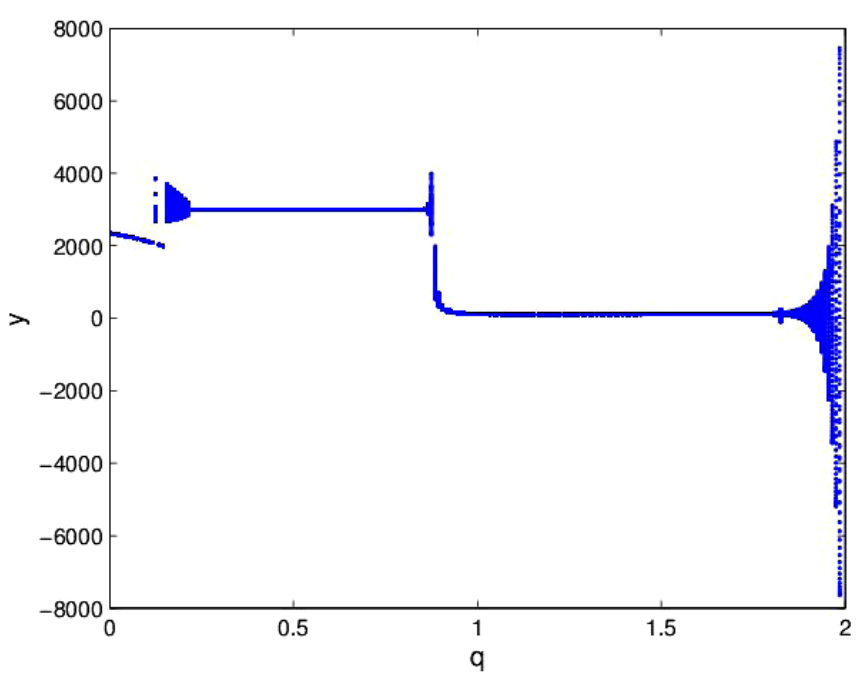

Fig. 2. Running state with the change of order $q$.

\section{Hopf Bifurcation of Fractional-Order IS-LM System}

\subsection{Hopf bifurcation of fractional-order system}

As we all know, Hopf bifurcation is obtained from linearization. So we should talk about the linearization theorem regarding fractional differential equation with Caputo derivative. As the result of $\mathrm{Li}$ and Ma's research [Zhang \& Wei, 2004], if all eigenvalues $\operatorname{eig}(A)_{i}$ of $\mathrm{A}$ satisfy: $|\operatorname{eig}(A)| \neq 0$, and $\left|\arg \left(\operatorname{eig}(A)_{i}\right)\right| \neq \pi q / 2$, then the origin of the linear system is called a hyperbolic equilibrium point. For this point, the vector field $f(x)$ is topologically equivalent with its linearization vector field $D^{q} f(0)$ in the neighborhood of the origin, so, the linearization in this paper is satisfied.

For a differential system, when Hopf bifurcation occurs, periodic solution or limit cycle will appear surrounding the equilibrium point, and it will arise or go away with the variation of the parameter. For Hopf bifurcation, we must study the stability of the equilibrium point, thus inevitably analyze the characteristic equation and eigenvalues of the system. For the integer-order system, the Hopf bifurcation existence conditions are:

(1) The eigenvalues near the equilibrium point of the system is in the form of $\lambda(\omega)=\alpha(\omega)+$ $i \beta(\omega)$.

(2) There is a pair of purely imaginary eigenvalues, which makes $\alpha(\omega)=0, \beta(\omega)>0$, and $\alpha^{\prime}(\omega) \neq 0$.

(3) The real parts of other eigenvalues are not equal to 0 .

The fractional-order system has the same equilibrium point, characteristic equation and eigenvalues with corresponding integer-order system, but the discrimination method of the stability of equilibrium point is totally different. We cannot use the Hopf bifurcation condition of the integer order, and we need to have a set of fractional-order Hopf bifurcation conditions and related ideas. There are two main methods in the process of calculating the Hopf bifurcation: Poincaré-Birkhoff canonical form method and successor function method. Since Poincaré-Birkhoff canonical form needs the center manifold theorem and vector inner product, so it is not suitable for the derivation process of Hopf bifurcation under the fractional-order condition. In this paper, we use successor function method to carry out a simple derivation. 
Theorem 1 [Existence of Hopf Bifurcation]. Assumption $q^{*}$ is the critical value of the order of the system when it enters into Hopf bifurcation near the equilibrium point, and $q^{*} \in(0,1)$, Hopf bifurcation occurs surrounding the equilibrium point when the following conditions hold:

(1) The characteristic equations of the original system has a pair of complex conjugate roots $\lambda_{1,2}=$ $\alpha+i \beta$, and the other eigenvalues are negative real roots.

(2) $m\left(q^{*}\right)=q^{*} \pi / 2-\min \left|\arg \left(\lambda_{i}\right)\right|=0$.

(3) $\left.\frac{d m(q)}{d q}\right|_{q=q^{*}} \neq 0$ (transversality condition).

Proof. (Successor Function Method). First of all, let the equilibrium point of the linear approximation equation be at the origin, and make the trigonometric substitution to the system, then have the power series expansion along a certain direction according to the conditions. Finally we define the successor function, and assess it in relation to the origin and periodic solutions.

Only the two-dimensional case is discussed, and the high dimensional system can be obtained in similar way as two-dimensional system, and the Hopf bifurcation.

Suppose $w \subset R^{2}, w$ is an open set $f^{q}: w \times$ $\left(-\lambda_{0}, \lambda_{0}\right) \rightarrow R^{2}, f^{q}(x, \lambda)$ is an analytic function of $x \in w, \lambda \times\left(-\lambda_{0}, \lambda_{0}\right)$.

For the equation: $\frac{d^{q} x}{d t^{q}}=f(x, \lambda)$.

For any $\lambda$, the equilibrium point is $O(0,0)$, and the derivative operator $D^{q} f(0, \lambda)$ of $f^{q}(x, \lambda)$ on $x=$ 0 can be denoted as $A^{q}(\lambda)$.

The eigenvalues of $A^{q}(\lambda)$ are a pair of conjugate complex numbers $\alpha(\lambda) \pm i \beta(\lambda), \beta(\lambda)>0$.

Note: Under the condition of integer order, the requirement is $\alpha(\lambda)=0$, at this moment, the operating state of the system would have changed; but under the condition of fractional order, the operating condition of the system would have changed into: $m_{i}(q)=q \pi / 2-\min \left|\arg \left(\pi_{i}\right)\right|=0$.

When $m_{i}(q)<0$, the equilibrium point is asymptotically stable. And when $m_{i}(q)>0$, the equilibrium point is no longer stable. So, the function $m_{i}(q)$ has the same effect as the real part of eigenvalues in integer-order systems.

At this moment, for sufficiently small $x$, there is only one analytic function $m_{i}(q)=q \pi / 2-$ $\min \left|\arg \left(\pi_{i}\right)\right|=0$, let the trajectory of the system pass through $(x, 0)$ as a close orbit, forming the stable period, and $\lim _{\lambda \rightarrow 0}[x(\lambda) / \sqrt[2 m]{\lambda}]=k \neq 0(m$ is a positive integer).

Obviously, under the fractional-order condition, the first condition is satisfied, eigenvalues of fractional-order systems are similar to the integerorder systems. For the second condition, the switching points of the stability, the order is:

$$
q *=\frac{2}{\pi} \arctan \left|\frac{\beta(\omega)}{\alpha(\omega)}\right|, \quad q * \in(0,1) .
$$

The third condition is transversality condition, it guarantees that the system running state will be changed when the system passes through the order $q *$, which is the Hopf bifurcation parameter. That is, the state of the system is different in $(0, q *)$ and $(q *, 1)$.

We can see that the existence conditions of the fractional Hopf bifurcation is improved based on the Hopf bifurcation condition of integer-order system, and combined with the discriminant conditions of equilibrium point stability of fractional-order system, then gives the exact proof. The existence condition of the Hopf bifurcation has corresponding advantages, for example, the order can be analyzed as the Hopf bifurcation parameter, it can satisfy all three conditions in Hopf bifurcation conditions in integer-order system. So this paper adopts these conditions within the Hopf bifurcation analysis of fractional-order IS-LM system.

It should be noted that, scholars have proved that, different to the integer-order systems, the periodic solutions or limit cycle of fractional-order system near Hopf bifurcation point may not be the solution set, but it indeed attracts the solution nearby [Tavazoei et al., 2009a; Tavazoei et al., 2009b].

\subsection{Numerical simulation of Hopf bifurcation in fractional-order system}

We can see that, after numerical calculation, the eigenvalues of system (3) are

$$
\begin{aligned}
\lambda_{1} & =-1.43660468, \\
\lambda_{2,3} & =0.51016306 \pm 1.64185723 i .
\end{aligned}
$$

This satisfies the first condition of Hopf bifurcation in Lemma 1, there exist a pair of pure imaginary conjugate eigenvalues, and other eigenvalues are negative real numbers, easy to verify, the third 
condition (transversality condition) is satisfied. So with the formula $m\left(q^{*}\right)=q^{*} \pi / 2-\min \left|\arg \left(\lambda_{i}\right)\right|=0$, we can get the order conditions of fractional-order IS-LM system (3) when Hopf bifurcation occurs.

$$
q^{*}=\frac{2 \min \left|\arg \left(\lambda_{i}\right)\right|}{\pi}=0.8042 .
$$

That is, when $q=0.8042$, the system (2) shows Hopf bifurcation.

From the three-dimensional fractional-order differential systems arises the limit cycle, as shown in Fig. 3.

As shown in Fig. 3, we know that, although the fractional-order system does not have an actual cycle solution or limit cycle, the limit cycles do exist in the phenomenon of attracting solutions around it. Figure 3(a) shows the limit cycle of system (2) under the order of $q^{*}=0.8042$, meanwhile, the system appears with a periodic solution

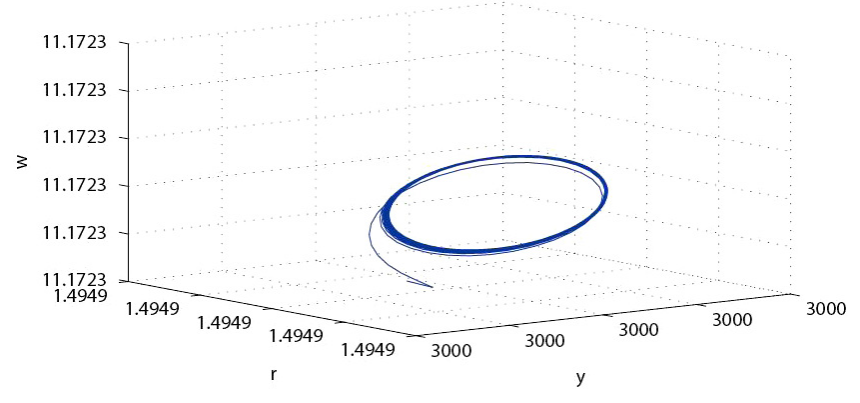

(a)

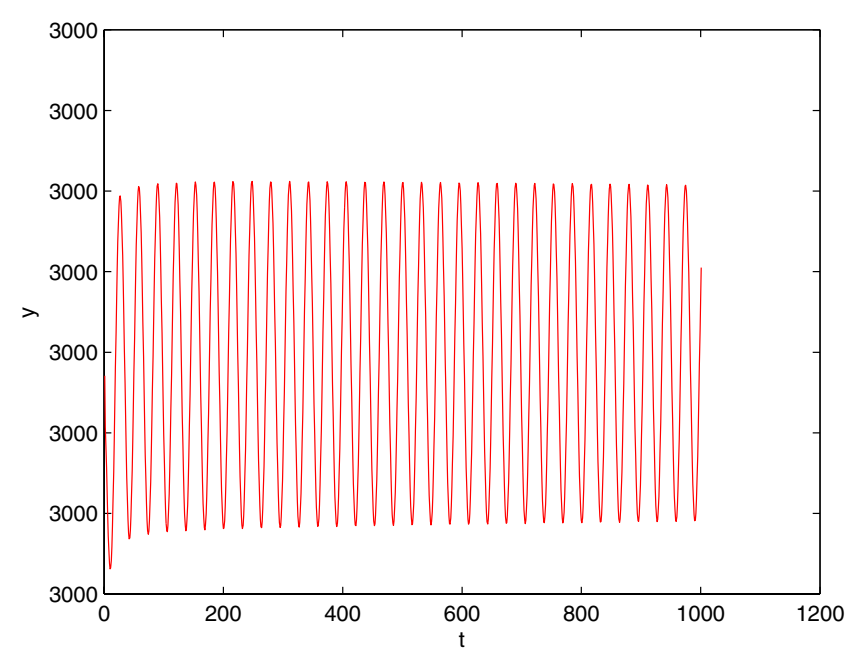

(b)

Fig. 3. Limit cycle and waveform of $y(t)$ in system (2). (a) Limit cycle in system (2) and (b) waveform of $y(t)$ in system (2). as shown in Fig. 3(c), $y(t)$ presents the periodic motion.

For the condition of the current parameters, considering the system running state nearby $q^{*}=$ 0.8042 , analyze the running state changes of the system when the order $q$ gradually passes through $q^{*}=0.8042$, as shown in Fig. 4 .

For a nonlinear system, once the Hopf bifurcation occurs, the system will change its stability, this change can be further determined by the first Lyapunov coefficient. In the economic system, Hopf bifurcation can cause the system to have a similar change. We can see that when the system reaches $q^{*}<0.8042=0.792$, variables of economic system converge, economic system stabilizes, variables become steady, which is the situation we would like to see. When the system reaches $q^{*}>0.8042=$ 0.81 , the variables of economic system diverge, the economic variables grow faster, the system cannot be controlled; when the system reaches $q^{*}=0.8042$, cyclical changes occur in the economic system. To ensure that macroeconomic control is stable, we should guarantee the order of the system is kept at $q^{*}<0.8042$, so that the economic system can be controlled, macroeconomic control mechanisms can proceed smoothly.

\subsection{Influence of the parameters on the fractional-order IS-LM system}

Both for the integer-order system and fractionalorder system, the parameter has considerable effect on the system dynamic evolution characteristics. In the study process of nonlinear dynamics, the parameter is usually used as a bifurcation parameter. In this paper, we choose $\alpha$ (rate of change of income $y(t))$ to study its bifurcation condition in different systems, to show the different influences of parameters on fractional- and integer-order systems, as shown in Fig. 5.

As shown in Fig. 5, with the variation of $\alpha$, the integer-order and the fractional-order systems show different operating conditions, when $q=1$, the system is an integer-order system, with the variation of $\alpha$, the system presents the divergent trend, $y(t)$ is discrete with the increase of $\alpha$ gradually, the corresponding Lyapunov exponent is greater than 0 , showing the same characteristics, as shown in Fig. 5(a). When $q=0.2$, the system is a fractionalorder system, when $\alpha \in(0,0.92)$, the system is 
Complexity and Hopf Bifurcation Analysis of Fractional-Order IS-LM Macroeconomic System

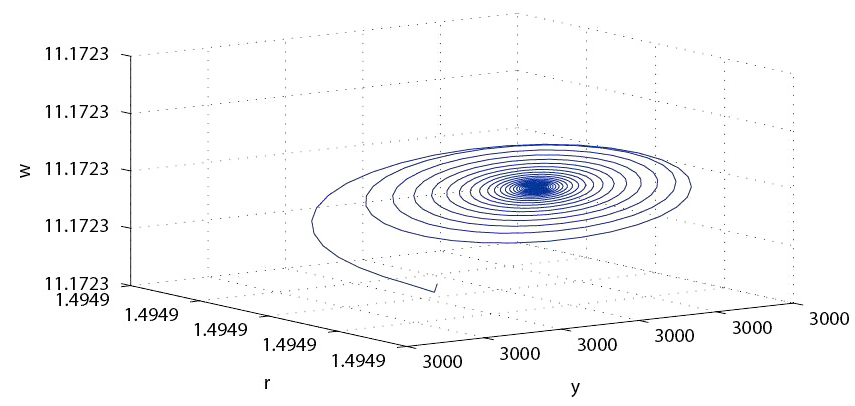

(a)

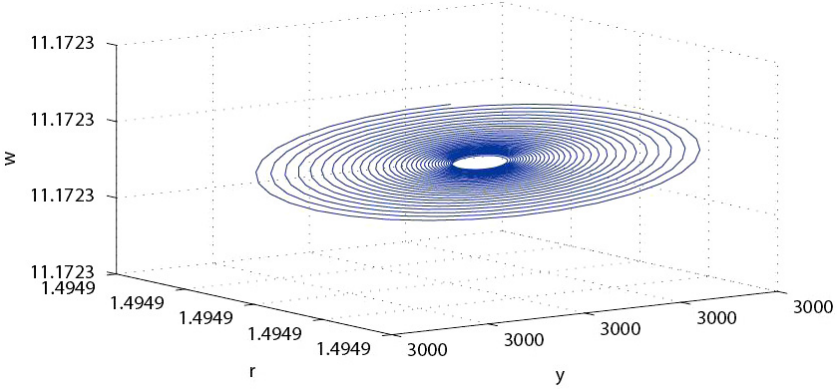

(c)

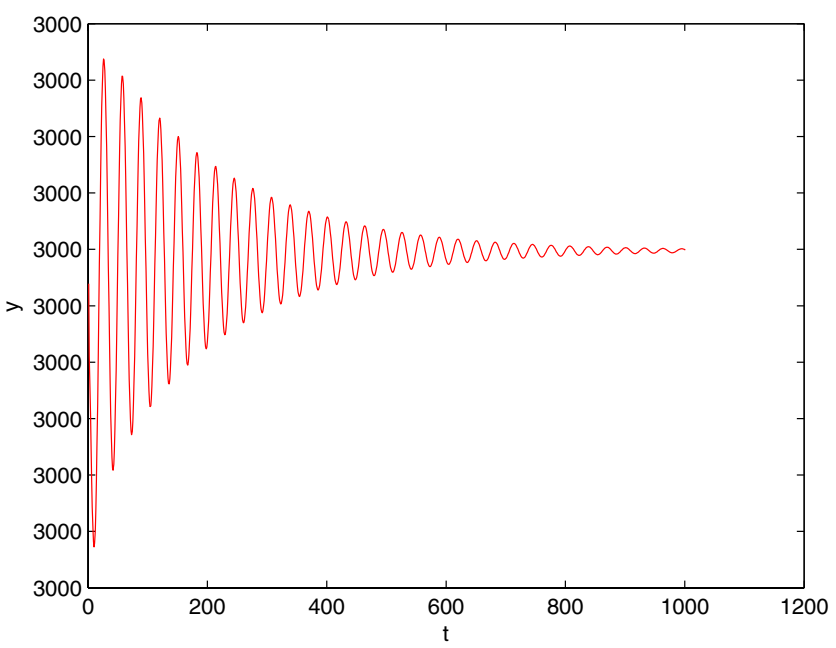

(b)

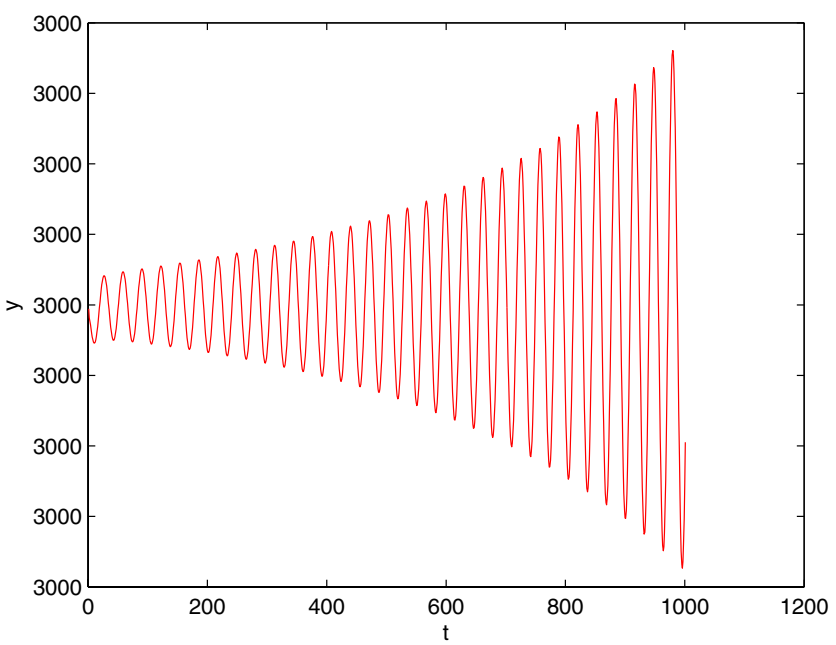

(d)

Fig. 4. The phase diagrams and waveform near $q^{*}=0.8042$. (a) Phase diagram when $q=0.792$, (b) waveform when $q=0.792$, (c) phase diagram when $q=0.81$ and (d) waveform when $q=0.81$.

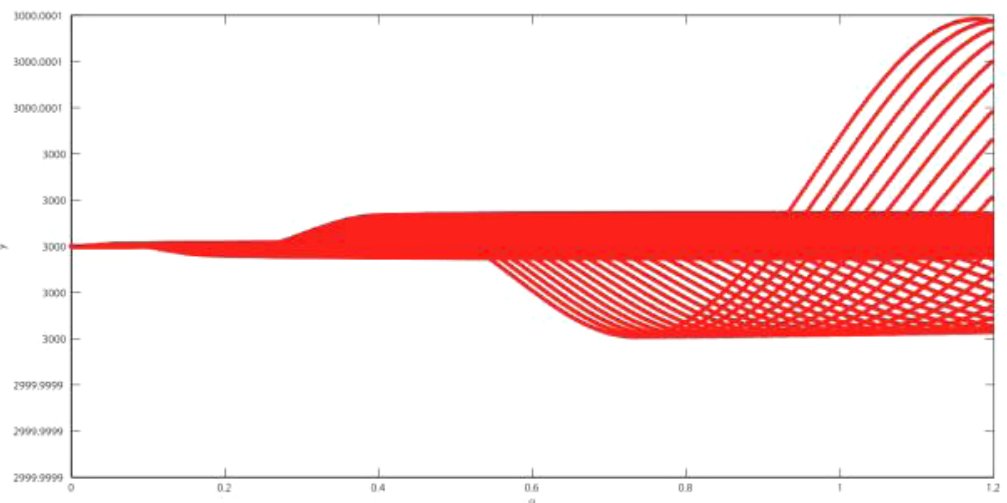

(a)

Fig. 5. Influence of $\alpha$ on integer-order and fractional-order systems. (a) Influence of $\alpha$ when $q=1$ and (b) influence of $\alpha$ when $q=0.2$. 


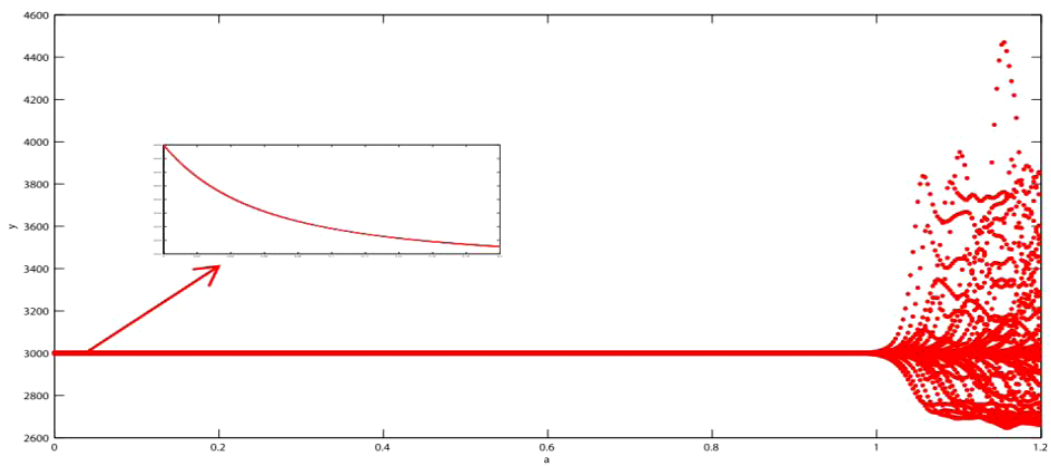

(b)

Fig. 5. (Continued)

approximately stable, and when $\alpha$ is larger than 0.92 , the system exhibits a chaotic state.

Therefore, the changes of the parameters cause different effects to different systems. In the actual application process, we should consider the state of the system and parameters, to achieve stable equilibrium state of the system.

\section{Conclusion}

In this paper, we introduced the fractional-order theory to deepen and complete the analysis of the IS-LM model, meet the memory characteristics of economic variables, and improve the analysis capabilities of the traditional economic model. Then, we focus on the stability condition and Hopf bifurcation conditions of the fractional-order system with theoretical proofs. We also analyze the complex dynamics characteristics of the system, such as bifurcations, strange attractor, limit cycle of the Hopf bifurcation, through phase diagrams and waveforms analyses.

Our research results show that the fractional orders have a significant influence on the running state of the system. With the change of the orders, the system will have different operating states, and the Hopf bifurcation is formed under a certain condition of the orders. In this paper, the economic system order is found to be $q=0.8042$, and at this order state, the system forms a periodic solution. Therefore, in the actual process of economic activities, we should ensure that the system meets the order condition $q^{*}<0.8042$, so as to ensure the macroeconomic state's stability and controllability. Furthermore, the changes of parameters can also help the system to enter to the asymptotic stability state. In the actual application process, we should properly choose the change ranges of the orders and the parameters, so as to ensure the operation of macroeconomic regulation and control is smooth.

\section{Acknowledgments}

The research was supported by the National Natural Science Foundation of China (Grant No. 61273231), and Doctoral Fund of Ministry of Education of China (Grant No. 20130032110073).

\section{References}

Ahmed, E., El-Sayed, A. M. A. \& El-Saka, H. A. A. [2006] "On some Routh-Hurwitz conditions for fractional-order differential equations and their applications in Lorenz, Rössler, Chua and Chen systems," Phys. Lett. A 358, 1-4.

Barbosa, R. S., Tenreiro Machado, J. A., Vinagre, B. M. \& Calderón, A. J. [2007] "Analysis of the van der Pol oscillator containing derivatives of fractional-order," J. Vibr. Contr. 13, 1291-1301.

Cai, J. [2005] "Hopf bifurcation in the IS-LM business cycle model with time delay," Electron. J. Diff. Eqs. 15, 1-6.

Cesare, L. D. \& Sportelli, M. [2005] "A dynamic IS-LM model with delayed taxation revenues," Chaos Solit. Fract. 25, 233-244.

Chen, J. H. \& Chen, W. C. [2008] "Chaotic dynamics of the fractionally damped van der Pol equation," Chaos Solit. Fract. 35, 188-198.

Chian, A. L., Rempel, E. L. \& Rogers, C. [2006] "Complex economic dynamics: Chaotic saddle, crisis and intermittency," Chaos Solit. Fract. 29, 1194-1218.

Gao, Q. \& Ma, J. H. [2009] "Chaos and Hopf bifurcation of a finance system," Nonlin. Dyn. 58, 209-216.

Ge, Z. M. \& Ou, C. Y. [2007] "Chaos in a fractionalorder modified Duffing system," Chaos Solit. Fract. 34, 262-291. 
Li, Y. X., Tang, W. K. S. \& Chen, G. [2005] "Generating hyperchaos via state feedback control," Int. J. Bifurcation and Chaos 15, 3367-3375.

Li, C. P. \& Ma, Y. T. [2013a] "Fractional dynamical system and its linearization theorem," Nonlin. Dyn. $\mathbf{7 1}, 621-633$.

Li, T. \& Ma, J. H. [2013b] "The complex dynamics of R\&D competition models of three oligarchs with heterogeneous players," Nonlin. Dyn. 72, 45-54.

Li, X. \& Wu, R. C. [2014] "Hopf bifurcation analysis of a new commensurate fractional-order hyperchaotic system," Nonlin. Dyn. 78, 279-288.

Liu, X. J., Hong, L. \& Yang, L. X. [2014] "Hopf bifurcations of a stochastic fractional-order van der Pol system," Abstr. Appl. Anal. 6, 1-10.

Lorenz, H. W. [1993] Nonlinear Economic Dynamics and Chaotic Motion (Springer, NY).

Lorenz, H. W. \& Nusse, H. E. [2002] "Chaotic attractors, chaotic saddles, and fractal basin boundaries: Goodwin's nonlinear accelerator model reconsidered," Chaos Solit. Fract. 13, 957-965.

Ma, J. H. \& Chen, Y. S. [2001a] "Study for the bifurcation topological structure and the global complicated character of a kind of nonlinear finance system (I)," Appl. Math. Mech. 22, 1240-1251.

Ma, J. H. \& Chen, Y. S. [2001b] "Study for the bifurcation topological structure and the global complicated character of a kind of nonlinear finance system (II)," Appl. Math. Mech. 22, 1375-1382.

Ma, S. J., Xu, W., Li, W. \& Fang, T. [2006] "Analysis of stochastic bifurcation and chaos in stochastic Duffing-van der Pol system via Chebyshev polynomial approximation," Chin. Phys. 15, 1231-1238.

Ma, J. H. \& Gao, Q. [2007] "Analysis and simulation of chaotic character of business cycle model," Dyn. Contin. Discr. Impuls. Syst. 1, 310-315.

Ma, J. H. \& Zhang, J. L. [2012] "Research on the price game and the application of delayed decision in oligopoly insurance market," Nonlin. Dyn. 52, 14791489

Mao, Y. B., Chen, G. \& Lian, S. G. [2004] "A novel fast image encryption scheme based on the $3 \mathrm{D}$ chaotic
Baker map," Int. J. Bifurcation and Chaos 14,36133624 .

Milhaela, N., Dumitru, O. \& Constantin, C. [2007] "Hopf bifurcation in a dynamic IS-LM model with time delay," Chaos Solit. Fract. 34, 519-530.

Oldham, K. \& Spanier, J. [1974] The Fractional Calculus (Academic Press).

Podlubny, I. [1998] Fractional Differential Equations: An Introduction to Fractional Derivatives, Fractional Differential Equations, to Methods of Their Solution and Some of Their Applications (Academic Press).

Ross, B. (ed.) [1975] The Fractional Calculus and Its Applications, Lecture Notes in Mathematics, Vol. 457 (Springer-Verlag, Berlin).

Schinasi, G. J. [1982] "Fluctuations in a dynamic, intermediate-run IS-LM model: Applications of the Poincaré-Bendixon theorem," J. Econ. Th. 28, 369375.

Sun, H., Chen, W. \& Chen, Y. [2009] "Variable-order fractional differential operators in anomalous diffusion modeling," Phys. A: Stat. Mech. Its Appl. 388, 45864592.

Szydlowski, M. \& Krawiec, A. [2005] "The stability problem in the Kaldor-Kalecki business cycle model," Chaos Solit. Fract. 25, 299-305.

Tavazoei, M. S., Haeri, M. \& Attari, M. [2009a] "A proof for nonexistence of periodic solutions in time invariant fractional-order systems," Automatica 8, 1886-1890.

Tavazoei, M. S., Haeri, M., Attari, M., Bolouki, S. \& Siami, M. [2009b] "More details on analysis of fractional-order van der Pol oscillator," J. Vibr. Contr. 6, 803-819.

Tu, H. L. \& Ma, J. H. [2014] "Stability and Hopf bifurcation analysis on a dynamic macro-economy system model with money supply delay," Nonlin. Dyn. 76, 497-508.

Zhang, C. R. \& Wei, J. J. [2004] "Stability and bifurcation analysis in a kind of business cycle model with delay," Chaos Solit. Fract. 22, 883-896. 